\title{
Review about Various Satellite Image Segmentation
}

\author{
Shabir Ahmed Mir, T. Padma. \\ Information Technology, AMET University, Chennai, India \\ Department of computer Applicaions, Sona College of Technology, Salem, India
}

\begin{tabular}{l}
\hline \hline Article Info \\
\hline Article history: \\
Received Nov 30, 2017 \\
Revised Jan 15, 2018 \\
Accepted Feb 4, 2018 \\
\hline Keywords: \\
EM \\
FCM \\
K-means \\
PSNR \\
Satellite Image segmentation
\end{tabular}

\begin{abstract}
In this paper, a review about different algorithm is proposed efficiently to segment the satellite images. Segmentation of Image is one of the promising and active researches in recent years. As literature prove that region segmentation will produce better results. Human visual perception is more effective than any machine vision systems for extracting semantic information from image. There are various segmentation techniques are available. Fuzzy C Means (FCM), Expectation Minimization (EM) and KMeans algorithm is developed to estimate parameters of the prior probabilities and likelihood probabilities. Finally Peak Signal to Noise Ratio (PSNR) is calculated for all the algorithms and reviewed.
\end{abstract}

Copyright $(0) 2018$ Institute of Advanced Engineering and Science. All rights reserved.

\section{Corresponding Author:}

Shabir Ahmed Mir, Information Technology, AMET University, Chennai, India.

\section{INTRODUCTION}

The study and the extraction of the different elements that compose an image constitute a fundamental task in the image processing and chain analysis. In fact, the necessity to replace the human observer by a computer for image analysis was in the origin of the development of image processing.

\section{BACKGROUND}

Survey about automatic detection \& classification of computer vision is presented in [1]. Bias, tiredness and loss of interest are the various factors influence the decision-making power of humaninspectors. Different rice grains are classified and identified automatically using machine vision system so these jobs need automation.

Automated separation of touching grains in digital images of thin sections is discussed in [2].Touching grain sections are separated using computer algorithm in binary images of granular material. Characteristic sharp contact wedges of touching grain sections in outline are detected using this algorithm. If the angle is smaller than user defined threshold value, intersection will create after checking.

Segmentation techniques and aggregate image processing algorithms for aggregate size and shape evaluation is described in [3]. Entity aggregate particle shape and size properties are analyzed and extracted using image processing and acquisition techniques. Digital single lens reflex camera is used to capture the aggregated images and these segmented images are fed into the validated university to calculate the particle shape and size for flat and elongated ratio.

Image processing based rice grading is explained in [4]. Length, shape, color, internal damage of rice is the features used to differentiate the rice grade using machine vision. Degree level of rice is analyzed and differentiated by histogram, RGB color model and edge detection etc. Machine vision system based efficient method for quality analysis of rice is presented in [5].Machine vision is used to assess the quality of 
the Indian basmati Oryza sativa L variety rice and Oryza sativa $L$ rice size is calculated using machine vision with chalky detection and broken rice.

Chalkiness in Rice: Potential for evaluation with image analysis is discussed in [6]. Image information processing is evaluated effectively in this method with inexpensive computer and chalkiness is categorized and measured using digital image scanner. Then method's feasibility is assessed.

Satellite and medical image segmentation based on multiple kernel fuzzy c-Means algorithm with ALS method is explained in [7]. Multiple kernel fuzzy c-means is used to generate initial contour curve during the curve as leaking at the boundary. Initial contour curve is generated using multiple kernel fuzzy cmeans during the curve propagation as leaking at the boundary. Finally different information's are combined using multiple kernel fuzzy c-means in segmentation algorithm. An outcome of periodized small side games with and without mental imagery on playing ability among intercollegiate level soccer players is also describes that [8]. Image Super Resolution Using Wavelet Transformation Based Genetic Algorithm explained in [9]. An integrated interactive technique for image segmentation using stack based seeded region growing and thresholding method is discussed in [10]. For analyzing the optimal performance of pest image segmentation is discussed as in [11]. Image segmentation based on doubly truncated generalized Laplace mixture model and $\mathrm{k}$ means clustering is discussed in [12].

\section{THE PROBLEM}

Among the various types of segmentation methods there may be more problems occurred in during the segmentation of the satellite images. Some of the problems are like data loss, mis-segmentation, etc. So in order to overcome this we will be stating some of the satellite image segmentation methods are discussed.

\section{PROPOSED SOLUTIONS}

\subsection{Expectation Maximization}

An iterative method is called as EM algorithm to discover maximum likelihood or maximum a posteriori parameters estimation in statistical models. Expectation step is performing by EM iteration alternatively and it creates a function using current estimate of the log-likelihood for the maximization step and parameters. Finally latent variable distribution is determined by these estimated parameters in the next expectation step.

The iteration of EM alternates between performing an Expectation (E) step, which creates a function for the expectation of the log-likelihood, evaluated using the current estimate for the parameters, and maximization (M) step, which computes parameters maximizing the predictable log-likelihood found on the E step. Distribution of the latent variables is determined by using this parameter in the next E step (Figure 1).

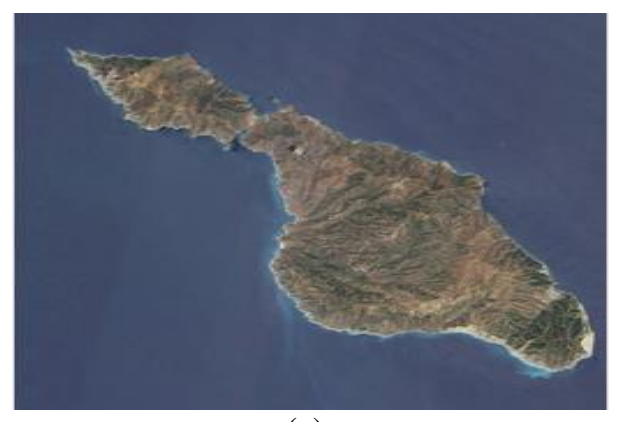

(a)

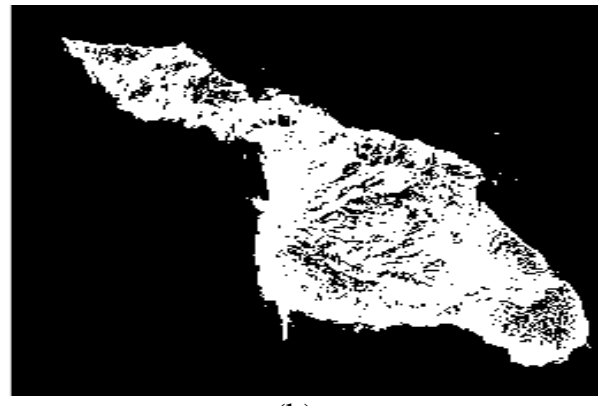

(b)

Figure 1. (a) Original image and (b) Segmented Image using EM algorithm

\subsection{Fuzzy C Means}

The method of clustering which allows one piece of data to belong to two or more clusters is called FCM. It is maximum used for pattern recognition. Cluster analysis involves assigning data points to clusters (also called buckets, bins, or classes), or homogeneous classes, such that items in the same class or cluster are as similar as possible, while items belonging to different classes are as dissimilar as possible (Figure 2). 


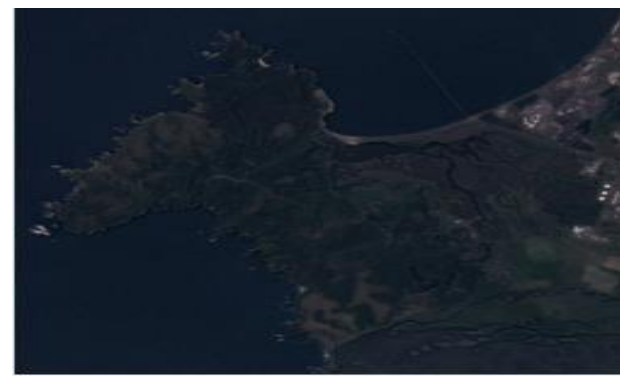

(a)

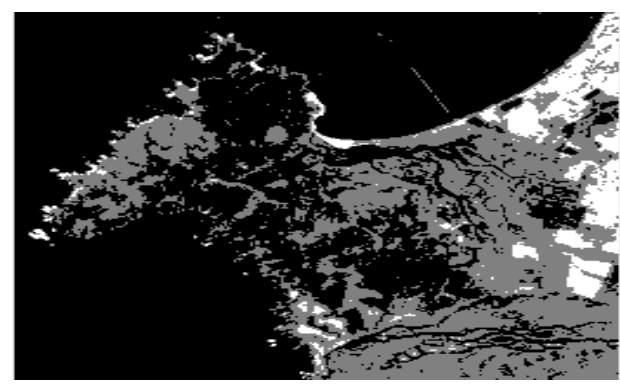

(b)

Figure 2. (a) Original image and (b) Segmented Image using FCM algorithm

\subsection{K-Means Clustering}

Vector quantization method is also called as k-means clustering, originally from signal processing, that is admired for cluster study in data mining. $\mathrm{K}$-means clustering is to panel $\mathrm{n}$ annotations into $\mathrm{k}$ clusters in which each observation belongs to the cluster with the bordering mean, helping as a prototype of the cluster. This outcome in a partitioning of the data space into Voronoi cells (Figure 3).

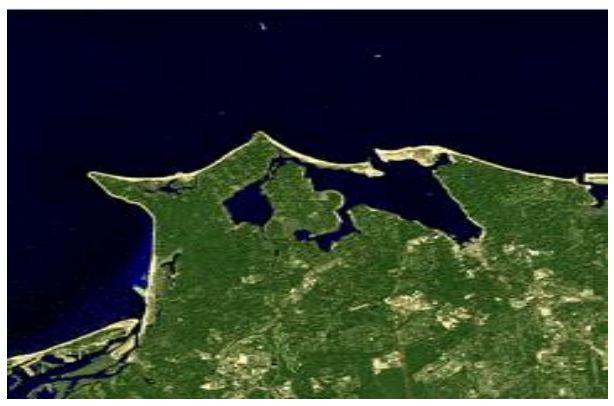

(a)

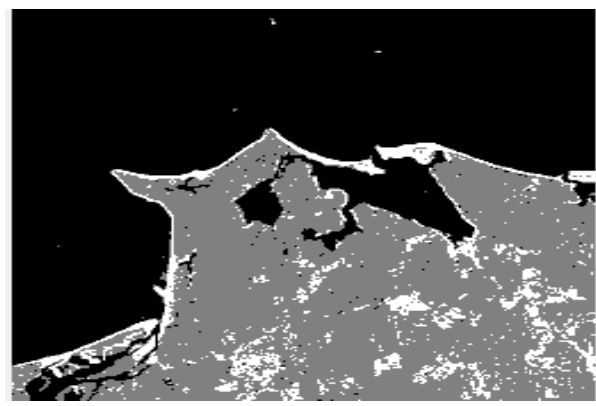

(b)

Figure 3. (a) Original image and (b) Segmented Image using K-Means algorithm

\section{RESULTS AND DISCUSSION}

Review of segmenting the satellite image using FCM, EM and K-Means is presented in this paper. From the investigational results the image segmentation using the proposed method was found to be more visually tempting than other existing algorithms. Figure 1,2,3 shows the proposed segmented image using EM, FCM and K-Means algorithm technique. The results show that this method is a very efficient optimization and obtained PSNR value is shown in Table 1.

\begin{tabular}{cc} 
Table 1. Comparison of Different techniques \\
\hline Method Used & PSNR Range \\
\hline EM & 38.65 \\
FCM & 36.23 \\
K-Means & 40.5 \\
\hline
\end{tabular}

\section{REFERENCES}

[1] Daljeet K. Automatic detection\& classification of rice using computer vision- a survey, International journal of innovative research in science, engineering and technology. 2015; 4-9.

[2] Van den Berg EH, Meesters AGCA, Kenter JAM, Schlager W. Automated separation of touching grains in digital images of thin sections, Computers \& geosciences. 2002; 28(2): 179-190.

[3] Moaveni M, Wang S, Hart J, Tutumluer E, Ahuja N. Aggregate size and shape evaluation using segmentation techniques and aggregate image processing algorithms, Revised Manuscript 13-4167 for Annual Meeting Compendium of Papers, 2013. 
[4] Tahir WPN, Hussin N, Htike ZZ, Naing WYN. Rice grading using image processing, ARPN Journal of Engineering and Applied Sciences. 2015; 10(21); 1-9.

[5] Birla R, Chauhan APS. An Efficient Method for Quality Analysis of Rice Using Machine Vision System. Journal of Advances in Information Technology. 2015; 6(3): 5-9.

[6] Yoshioka Y, Iwata H, Tabata M, Ninomiya S, Ohsawa R. Chalkiness in rice: potential for evaluation with image analysis, Crop Science. 2007; 47(5), 2113-2120.

[7] Yugander P, Sheshagiri BJ, Sunanda K, Susmitha E. Multiple kernel fuzzy C-means algorithm with ALS method for satellite and medical image segmentation, IEEE International Conference on Devices, Circuits and Systems. 2012; 244-248.

[8] Kumar AM, An outcome of periodized small side games with and without mental imagery on playing ability among intercollegiate level soccer players. Indian Journal of Science and Technology. 2015; 8(36): 15-21.

[9] Panda SS, Jena G, Image Super Resolution Using Wavelet Transformation Based Genetic Algorithm. In Computational Intelligence in Data Mining. 2016; 2: 355-361. Springer India.

[10] Hore S, Chakraborty S, Chatterjee S, Dey N, Ashour AS, Van Chung L, Le DN. An Integrated Interactive Technique for Image Segmentation using Stack based Seeded Region Growing and Thresholding. International Journal of Electrical and Computer Engineering. 2016; 6(6): 2773-2778.

[11] Sangari S, Saraswady D. Analyzing the Optimal Performance of Pest Image Segmentation using Non Linear Objective Assessments. International Journal of Electrical and Computer Engineering. 2016; 6(6): 2789-2793.

[12] Jyothirmayi T, Rao KS, Rao PS, Satyanarayana C. Image Segmentation Based on Doubly Truncated Generalized Laplace Mixture Model and K Means Clustering. International Journal of Electrical and Computer Engineering. 2016; 6(5): 2188. 